\title{
Characteristics of pregnant and lactating women with leprosy
}

\author{
Paula Sacha Frota Nogueira ${ }^{[1],[2], ~ E s c o l a ́ s t i c a ~ R e j a n e ~ F e r r e i r a ~ M o u r a ~}{ }^{[2]}$, \\ Andrezza Alves Diass ${ }^{[2]}$, Camila Félix Américo ${ }^{[2]}$, Lidiane Rebouças Aguiar ${ }^{[2]}$ \\ and Mayenne Myrcea Quintino Pereira Valente ${ }^{[2]}$
}

[1]. Departamento de Enfermagem, Universidade Federal do Ceará, Fortaleza, CE. [2]. Programa de Pós-Graduação em Enfermagem, Universidade Federal do Ceará, Fortaleza, CE.

\begin{abstract}
Introduction: The clinical characteristics of women who conceive during leprosy and the association between leprosy and pregnancy are not well known. Methods: This cross-sectional study included 49 pregnant or lactating women diagnosed with leprosy in 2011. Results: The patients had a clinical dimorphous form of leprosy (44.9\%), no physical incapacity at diagnosis $(87.5 \%)$, and no complications in either the patient or infant (33.4\%). In 36.3\% of cases, leprosy symptoms were presented in the last trimester of pregnancy, and in $31.9 \%$ of patients were in the first trimester of lactation. Conclusions: The association between leprosy and pregnancy should be routinely investigated, particularly in endemic areas.
\end{abstract}

Keywords: Leprosy. Infectious pregnancy complications. Public Health.

Leprosy has a complex spectrum of clinical presentations and severities. Conditions that affect the balance between Mycobacterium leprae infection and immunity, such as hormonal changes during puberty and pregnancy, expose patients to complications ${ }^{(1)}$.

High levels of steroids, thyroid hormones, and estrogen during pregnancy decrease cellular immunity, which reverses around the $12^{\text {th }}$ week after childbirth. The period of immunosuppression between the last trimester of pregnancy and the first 3 months after childbirth increases predisposition to infection with Mycobacterium leprae and the reactivation of M. leprae in women who were apparently cured ${ }^{1}$. Complications in children born to women with leprosy include lower birth weight, smaller placenta, slow growth, increased incidence of infection, and mortality in childhood ${ }^{(2)}$.

Pregnant women with leprosy should be closely monitored for anemia and uterine height, because the antibacterial drug dapsone, used in the treatment of leprosy, can cause hemolytic anemia ${ }^{(2)}$. This, together with the physiological anemia that develops during pregnancy, can have serious direct consequences for the mother and indirect consequences for the child, because it decreases the blood supply necessary for proper placental development ${ }^{(2)}$.

\footnotetext{
Address to: Profa. Paula Sacha Frota Nogueira. Depto. Enfermagem/UFC. R. Alexandre Baraúna 1115/Sala 05, Rodolfo Teófilo, 60430-160 Fortaleza, CE, Brasil. Phone: 5585 3366-8461

e-mail: sachanogueira@yahoo.com.br

Received 27 June 2014

Accepted 5 November 2014
}

Some characteristic associations between leprosy and pregnancy were reported in a retrospective study from the Carajás region of Brazil; detection rates of women with leprosy were 4.7 in 2007, 9.4 in 2008, and 4.3 in 2009, indicating the endemicity of this condition ${ }^{(3)}$.

The objective of the present study was to describe the clinical, demographic, and socioeconomic characteristics of women who were diagnosed with leprosy during pregnancy and/or lactation.

This descriptive cross-sectional study of 49 women with leprosy was performed by evaluating reported cases or medical records of pregnant and/or lactating women who presented with the signs and symptoms of leprosy, which included leprosy reactions, and/or recurrence on follow-up to a dermatology referral center in Fortaleza, Ceará, Brazil, between March and October 2011.

Data were collected through interviews, which were conducted by completing a form that included questions about participants' demographic and socioeconomic characteristics, as well as the clinical features of leprosy. Data relating to the clinical aspects of leprosy were obtained from the medical records of each participant and were supplemented with the interviews.

Data were processed using the Statistical Package for Social Sciences, Version 17.0. Descriptive statistics and bivariate analysis to verify the statistical association between the leprosy reaction onset or diagnosis and pregnancy and/or lactation were performed using the chi-square test, chi-square tests with continuity correction, and chi-square trend. All analyses were considered statistically significant if $\mathrm{p}<0.05$. 
The Ethics Committee in Research of the Center of Dermatology Dona Libânia approved the study (protocol number 012/2011). All women provided informed consent before data collection.

The 49 women who participated in this study had a mean age of $32.1 \pm 8.85$ years; most women were aged between 21 and 34 years $(22-44.9 \%)$, were residents of Fortaleza-CE (37-75.5\%), had an average level of education of $7.85 \pm 3.18$ years of study, and had a regular partner (40-81.6\%). The average family income was $\mathrm{R} \$ 840.59 \pm \mathrm{R} \$ 554.04$, with an average number of 4.97 people per household, resulting in an average per capita income of $\mathrm{R} \$ 168.80$.

Leprosy is prevalent in adult and elderly populations, a fact that can be attributed to increased social contact and the long incubation period of leprosy. The average patient age of 32 years in this study is consistent with the findings of a study conducted in $2011^{(4)}$. This particular average age may also be associated with the methodological boundaries of working with women who were both of childbearing age and were legally capable to sign the informed consent, resulting in women between 18 and 49 years being evaluated.

Education level is an indirect indicator of general social condition, and the results of this study reflected education's relevance in controlling the disease. In addition, leprosy has traditionally been associated with unfavorable socioeconomic conditions, including low income. Level of knowledge about the disease, access to health services, and understanding of the guidelines for the treatment and prevention of leprosy are linked to the ability to provide self-care and the number of years of education ${ }^{(5)}$.

The clinical characteristics of patients with the predominant clinical form of leprosy are borderline leprosy (22-44.9\%), use of multidrug therapy for multibacillary forms (23-57.5\%), smear negative (32-65.3\%), no physical incapacity at diagnosis (35-87.5\%), and previous contact with patients with leprosy (30-61.3\%), most of whom were relatives (27-55.1\%). Leprosy reactions occurred in $30.6 \%$ of participants, $60 \%$ of whom were pregnant and/or lactating at the diagnosis of the reaction.

The clinical features of leprosy in this study group were similar to another study in which 191 (94\%) of 203 total women evaluated were classified as having multibacillary leprosy. There was a significant association between multibacillary classification and female sex, with women being 4 times more likely to be classified as having multibacillary disease than men [odds ratio $=4.4,(95 \% \text { confidence interval, 2.22-9.05), } \mathrm{p}<0.05]^{(5)}$.

Most signs of leprosy among pregnant and/or lactating women in this study occurred during the last trimester of pregnancy and the 3 months after childbirth [10 (20.4\%) women]. Eight (16.3\%) women did not remember when they noticed the first sign of the disease. The proportion of women who first showed signs of leprosy during each period (i.e., the last trimester of pregnancy and the 3 months after childbirth) was considered statistically nonsignificant $(p=0.335)$.

The findings of this study were consistent with published reports showing that the emergence of leprosy during pregnancy and/or lactation most commonly occurs in the last trimester of pregnancy and the 3 months after childbirth ${ }^{(1)(2)(4)}$; however, it is worth noting that these data were subject to recall bias from the study participants.

During the assessment and evaluation of complications resulting from leprosy and pregnancy, we excluded detection of early signs and symptoms, which was a criterion for inclusion in our sample (Table 1).

Twenty-four (48\%) women reported no complications; this may be explained by the cross-sectional design of the study. At the time of interviews, $5(10 \%)$ women were pregnant, and $5(10 \%)$ were breastfeeding. The most frequently reported complications were low birth weight in newborns (12-22.3\%) and leprosy reactions in women (10-18.6\%) (Table 1).

Based on the interviews and analysis of clinical data from the medical records, leprosy reactions during pregnancy or lactation were indicative of a definitive diagnosis in 11 (22\%) women. Demand for care in the reference center was also a deciding factor in diagnosis, which was also pointed out in a review of clinical cases about this association ${ }^{(4)}$.

Complications arising from concomitant pregnancy and leprosy can be avoided by providing guidance to women with leprosy regarding the importance of postponing pregnancy and using appropriate contraception. Follow-up with these patients regarding quality family planning is necessary and consistent with the standard of care for leprosy.

TABLE 1 - Reported complications related to pregnancy and leprosy events.

\begin{tabular}{lcc}
\hline Variables $(\mathrm{n}=49)$ & Number & Percentage \\
\hline Mother and baby without complications & 18 & 33.4 \\
Newborn with low birth weight $(<2,000 \mathrm{~g})$ & 12 & 22.3 \\
Emergence of leprosy reaction & 10 & 5.6 \\
Aggravation of leprosy toward the lepromatous pole & 3.2 \\
Relapse & 3.5 & 3.5 \\
Increased childhood infections & 3.5 \\
Other (aggravation of leprosy reaction, exfoliative dermatitis in the infant, premature newborn) & 3 \\
\hline
\end{tabular}




\section{CONFLICT OF INTEREST}

The authors declare that there is no conflict of interest.

\section{REFERENCES}

1. Duncan E. Leprosy in pregnancy. In: Nunzi E, Massone C, editors. Leprosy: a practical guide. Milan, Italy: Springer; 2012. p. 331-340.

2. Duncan ME. The A9 study: the longest cohort study in the history of leprosy-an overview. Ethiop Med J 2007; 45 (suppl I):1-7.
3. Palácios VRCM, Bichara CNC, Dias RS, Sousa Jr AS, Cardoso RF, Barreiros GVN, et al. Analysis of the detection coefficient for the association between leprosy and pregnancy in the integration region of Carajás, State of Pará, Brazil. Rev Soc Bras Med Trop 2013; 46:650-653.

4. Nogueira PSF, Moura ERF, Oriá MOB, Moreira LPM, Dias AA. Consequences of the interaction between leprosy and pregnancy. Rev Enferm UFPE 2012; 6:2242-2248.

5. Miranzi SSC, Pereira LHM, Nunes AA. Perfil epidemiológico da hanseníase em um município brasileiro, no período de 2000 a 2006. Rev Soc Bras Med Trop 2010; 43:62-67. 